\title{
Multifokale neuroendokrine Lungentumoren: Entstehung, Diagnostik und Behandlung
}

\author{
Multifocal Pulmonary Neuroendocrine Tumours: Genesis, Diagnostics and \\ Treatment
}

Autoren

Institute
A. Kirschbaum ${ }^{1}$, B. Beutel ${ }^{2}$, A. Rinke ${ }^{3}$, P. Rexin ${ }^{4}$, L. Fink ${ }^{5}$, R. Koczulla ${ }^{2}$, D. K. Bartsch ${ }^{1}$

Die Institutsangaben sind am Ende des Beitrags gelistet. eingereicht 15.9 .2015 akzeptiert nach Revision 29.11.2015

\section{Bibliografie}

DOI http://dx.doi.org/ 10.1055/s-0041-110291 Pneumologie 2016; 70: 123-129

(c) Georg Thieme Verlag KG Stuttgart · New York ISSN 0934-8387

\section{Korrespondenzadresse PD Dr. Andreas Kirschbaum} Klinik für Viszeral-, Thorax- und Gefäßchirurgie Universitätsklinikum Gießen und Marburg GmbH, Standort Marburg

Baldingerstraße

35033 Marburg

akirschb@med.uni-marburg.de

\section{Zusammenfassung \\ $\nabla$}

Selten werden multifokale neuroendokrine Lungentumoren diagnostiziert. Im Thorax-CT zeigen sich meist multiple Lungenherde unterschiedlicher Größe. Es ist anzunehmen, dass sich multifokale neuroendokrine Lungentumoren ursprünglich aus diffusen idiopathischen pulmonalen neuroendokrinen Zellhyperplasien (DIPNECH) entwickeln. Darunter versteht man Zellverbände von physiologisch im Bronchialsystem vorhandenen neuroendokrinen Zellen, die wachsen. Durchbrechen die Zellvermehrungen die bronchiale Basalmembran, so liegt bei einer Größe $\leq 5 \mathrm{~mm}$ ein Tumorlet, ab einer Größe von $5 \mathrm{~mm}$ ein Karzinoidtumor vor. Die Proliferationsgeschwindigkeit der Zellhyperplasien scheint unterschiedlich zu sein. Da ein nicht unerheblicher Teil der Patienten völlig asymptomatisch ist, werden die multifokalen neuroendokrinen Lungentumoren zufällig diagnostiziert. Daneben gibt es jedoch Patienten, die über Luftnot, Einschränkung der Leistungsfähigkeit und Husten klagen. Die Lungenfunktion kann dabei schon eingeschränkt sein. In diesen Fällen zeigt eine HR-CT-Thorax-Untersuchung häufig außer den Lungenherden zusätzlich noch eine peribronchiale Fibrose oder Bronchiektasen. Eine Bronchoskopie ist in der Regel nicht wegweisend. Die chirurgische thorakoskopische Lungenbiopsie gilt als diagnostischer Goldstandard. Typischerweise zeigt sich histologisch eine Mischung aus Zellhyperplasien, Tumorlets und Karzinoidtumoren. Über die Behandlung multifokaler neuroendokriner Tumoren gibt es keinen Konsens. Ausgehend von der Klinik und den Befunden im HRCT des Brustkorbes entwickelten wir ein stufenweises Vorgehen, das sich an dem Erfolg der einzelnen therapeutischen Maßnahmen orientiert. Die günstigste Prognose haben Betroffene ohne klinische Beschwerden, bei denen alle Lungenherde unter $5 \mathrm{~mm}$ sind. In diesen Fällen liegt das 5-Jahres-Überleben über 90\%.

\section{Abstract \\ $\nabla$}

Multifocal neuroendocrine lung tumour is a rare diagnosis. Multiple lung foci of different sizes are usually apparent on chest CT scans. It is assumed that multifocal neuroendocrine lung tumours originally develop from diffuse idiopathic pulmonary neuroendocrine cell hyperplasia (DIPNECH). This results in cell aggregations formed by proliferation of neuroendocrine cells that are already physiologically present in the bronchial system. If these cell proliferations break through the bronchial basement membrane, they are considered to constitute tumourlets if they measure $\leq 5 \mathrm{~mm}$ and carcinoid tumours if they are larger than $5 \mathrm{~mm}$. The speed of proliferation of the cell hyperplasias appears to vary. Many of the patients are completely asymptomatic, the multifocal neuroendocrine lung tumours being diagnosed by chance. However, other patients complain of breathlessness, reduced physical capacity and cough. There may also be reduction of lung function. In these cases, chest HRCT often reveals peribronchial fibrosis or bronchiectasis in addition to the lung foci. Bronchoscopy is usually not helpful. Surgical lung biopsy is considered to be the diagnostic gold standard. Histological examination typically shows a mixture of cell hyperplasias, tumourlets and carcinoid tumours. There is no consensus on the treatment of multifocal neuroendocrine tumours. Taking the clinical situation and the chest HRCT findings as our starting point, we developed a stepwise approach that is guided by the success of the individual therapeutic procedures. The most favourable prognosis is found in affected people without clinical symptoms whose lung foci all measure less than $5 \mathrm{~mm}$. In these cases the 5 -year survival rate is over $90 \%$. 


\section{Hintergrund \\ $\nabla$}

Eine diffuse idiopathische neuroendokrine Zellhyperplasie (DIP$\mathrm{NECH}$ ) ist definiert als eine generalisierte multifokale Proliferation pulmonaler neuroendokriner Zellen [1,2]. Charakteristisch sind in der Bildgebung multiple pulmonale Herde, bestehend aus neuroendokrinen Zellen, die kleiner als $5 \mathrm{~mm}$ sind. Diese werden auch als Tumorlets bezeichnet. Die DIPNECH wurde erstmalig ausführlicher 1992 bei 6 Patienten beschrieben. Alle Betroffenen waren Nichtraucher ohne Hinweis auf eine sonstige Lungenerkrankung [2]. Insgesamt wurden in der medizinischen Literatur weniger als 100 Fälle einer DIPNECH beschrieben [3]. Eine DIPNECH wird als präinvasive Läsion für die Entwicklung von Karzinoidtumoren gesehen [4]. Dabei sind Karzinoidtumoren als Ansammlungen von neuroendokrinen Zellen definiert, die größer als $5 \mathrm{~mm}$ sind. Die Inzidenz einer DIPNECH bei chirurgisch entfernten Karzinoidtumoren liegt bei 4,5\% [4]. Bei peripher gelegenen Karzinoidtumoren, die chirurgisch entfernt wurden, fand sich bei $76 \%$ der Fälle im peritumoralen Gewebe eine neuroendokrine Zellhyperplasie [5]. In einer Serie von 1090 Fällen von Bronchialkarzinomen wurde eine DIPNECH im dem Tumor angrenzenden sowie im gesunden Lungengewebe nur in $0,3 \%$ gefunden [6]. Mireskandari [7] beschrieb eine Serie von 4 Patienten, bei denen ein nicht kleinzelliges Lungenkarzinom zusammen mit einer DIPNECH auftrat. Es ist anzunehmen, dass sich die selten diagnostizierten multifokalen neuroendokrinen Lungentumoren ausgehend von einer DIPNECH entwickelt haben. Typischerweise zeigen sich in der Bildgebung mehr als ein Karzinoidtumor auf einer Seite oder mehr als ein Karzinoidtumor auf beiden Seiten, sowohl peripherer oder auch zentraler Lokalisation. Begleitend können in der Bildgebung auch multiple kleine pulmonale Herde $<5 \mathrm{~mm}$ nachweisbar sein. Eine pulmonale Fernmetastasierung eines extrathorakalen neuroendokrinen Tumors liegt definitionsgemäß nicht vor. Die multifokalen neuroendokrinen Lungentumoren treten in $80 \%$ bei Frauen im mittleren Lebensalter (durchschnittlich 58 Jahre) ohne Raucheranamnese auf [8]. Die Hälfte der Patienten sind völlig symptomlos, haben eine normale Lungenfunktion, und die Diagnose ist ein radiologischer Zufallsbefund [9]. Die andere Hälfte der Patienten weist Symptome wie Husten (70\%), Luftnot (63\%) und Abgeschlagenheit (25\%) auf [5]. Zusätzlich können verschiedene Lungenerkrankungen wie COPD, Lungenfibrose und Bronchiektasen simultan auftreten [10].

Ziel dieser Arbeit ist es, anhand eigener Fälle und einer Literaturrecherche die Pathophysiologie und Diagnostik bei multifokalen neuroendokrinen Lungentumoren darzustellen sowie ein stufenweises therapeutisches Vorgehen vorzuschlagen.

\section{Pathophysiologische Hypothese der Entwicklung von multifokalen pulmonalen Karzinoidtumoren aus neuroendokrinen Zellhyperplasien}

Feyrter beschrieb 1938 ein diffuses System von klaren Zellen im Epithel des Respirationstraktes [11]. Er fasste es als einen Teil eines Systems von diffusen neuroendokrinen Zellen auf (neuroendokrin aufgrund der Produktion Neuronen-spezifischer Enolase und neuropeptidenthaltender Zellgranula), die im gesamten Körper verteilt sind [11]. Weitere Untersuchungen zeigten, dass pulmonale neuroendokrine Zellen (sog. Kulchitsky-Zellen) physiologischerweise einzeln oder als Zellcluster in der Bronchuswand vorkommen und Neuroamine und -peptide synthetisieren können [9]. Diese Zellen spielen als Chemorezeptoren zur Erkennung von Hypoxie und beim Wachstum und der Regeneration von bronchialen Epithelzellen eine Rolle [9]. Die neuroendokrinen pulmonalen Zellen setzen Bombesin und Bombesin-ähnliche Peptidhormone frei, die über Fibroblasten induzierte Chemotaxis zu einer Bronchokonstriktion führen können [2,8]. Diese Vorgänge können eine peribronchiale und interstitielle Fibrose verursachen [12]. Die reaktiven neuroendokrinen Zellhyperplasien, assoziiert mit einer Lungenerkrankung, werden als eine Reaktion auf eine chronische Hypoxie und eine Lungenschädigung interpretiert [9]. Eine pulmonale neuroendokrine Zellhyperplasie (PNECH) wurde zuerst bei Personen diagnostiziert, die in großer Höhe lebten, als mögliche Reaktion auf die chronische Hypoxie [13].

Es gibt drei Arten der Proliferation neuroendokriner Zellen: 1. Reaktive pulmonale neuroendokrine Zellhyperplasie (PNECH), 2. Zellproliferation in Kombination mit Karzinoidtumoren und 3. die diffuse neuroendokrine Zellhyperplasie (DIPNECH) [9]. Die DIPNECH wird als diffuse Form der PNECH angesehen [9].

Es wurde diskutiert, ob es grundsätzliche Unterschiede zwischen der reaktiven Natur der PNECH-Proliferation als Reaktion auf pulmonale Verletzungen und der eher präneoplastischen Natur der DIPNECH gibt [14]. Jedoch gibt es darauf bisher keine eindeutige Antwort. Eine DIPNECH gilt als präinvasive Läsion von typischen Karzinoidtumoren [15]. Miller et al. [16] untersuchten 25 Patienten nach Resektion von peripheren Karzinoidtumoren. In $76 \%$ der Fälle lag zusätzlich eine DIPNECH vor. Bei bis zu $42 \%$ dieser Patienten zeigte sich jedoch auch zusätzlich eine obliterative Bronchiolitis [17,18].

Die lokalen neuroendokrinen Zellhyperplasien sind eine Ansammlung von Zellnestern, die jedoch die bronchiale Basalmembran respektieren. Durchbrechen die Zellen durch ihr Wachstum die Basalmembran und ist ihre Größe $\leq 5 \mathrm{~mm}$, so liegt ein sog. Tumorlet vor. Ab einer Größe von über $5 \mathrm{~mm}$ wird das Tumorlet zum Karzinoidtumor. Je nach Höhe der Mitoserate liegt ein typischer (<2 Mitosen pro $\mathrm{mm}^{2}$ ) oder ein atypischer ( $>2$ Mitosen pro $\left.\mathrm{mm}^{2}\right)$ Karzinoidtumor vor.

Wie lange es dauert, bis sich aus einer neuroendokrinen Zellhyperplasie ein Karzinoidtumor entwickelt, ist nicht bekannt. Ebenfalls scheint möglicherweise eine unterschiedliche Proliferationsgeschwindigkeit der einzelnen betroffenen neuroendokrinen Zellen vorzuliegen. Dies erklärt das typische histologische Nebeneinander aus Tumorlets und Karzinoidtumoren.

Fallbericht 1: Eine 59-jährige Patientin klagte seit mehr als einem Jahr über einen zunehmenden Husten und zuletzt zunehmende Hämoptysen. Die Lungenfunktion war mäßiggradig eingeschränkt (FEV1 67\%). Eine Computertomografie des Thorax zeigte beidseitige pulmonale Rundherde verschiedener Größe und einen ca. $3 \mathrm{~cm}$ großen zentralsitzenden Tumor im rechten Unterlappen. Die bronchoskopischen Biopsien des Tumors ergaben einen neuroendokrinen Tumor. Es erfolgte eine untere Bilobektomie mit radikaler mediastinaler Lymphadenektomie. Die histologische Untersuchung zeigte in beiden Lappen multiple neuroendokrine Tumoren. Der größere Tumor im Unterlappen war ein atypischer neuroendokriner Tumor mit einer Ki67 Proliferationsrate von $22 \%$. Daneben zeigte sich ein weiterer atypischer Karzinoidtumor (Größe: 0,6 cm) im Mittellappen (Ki67 Proliferationsrate $18 \%$ ). In beiden Lappen wurden noch > 10 weitere typische Karzinoidtumoren (Ki67 Proliferationsrate $<1 \%$ ), Tumorlets und eine DIPNECH histologisch nachgewiesen (siehe - Abb.1). 


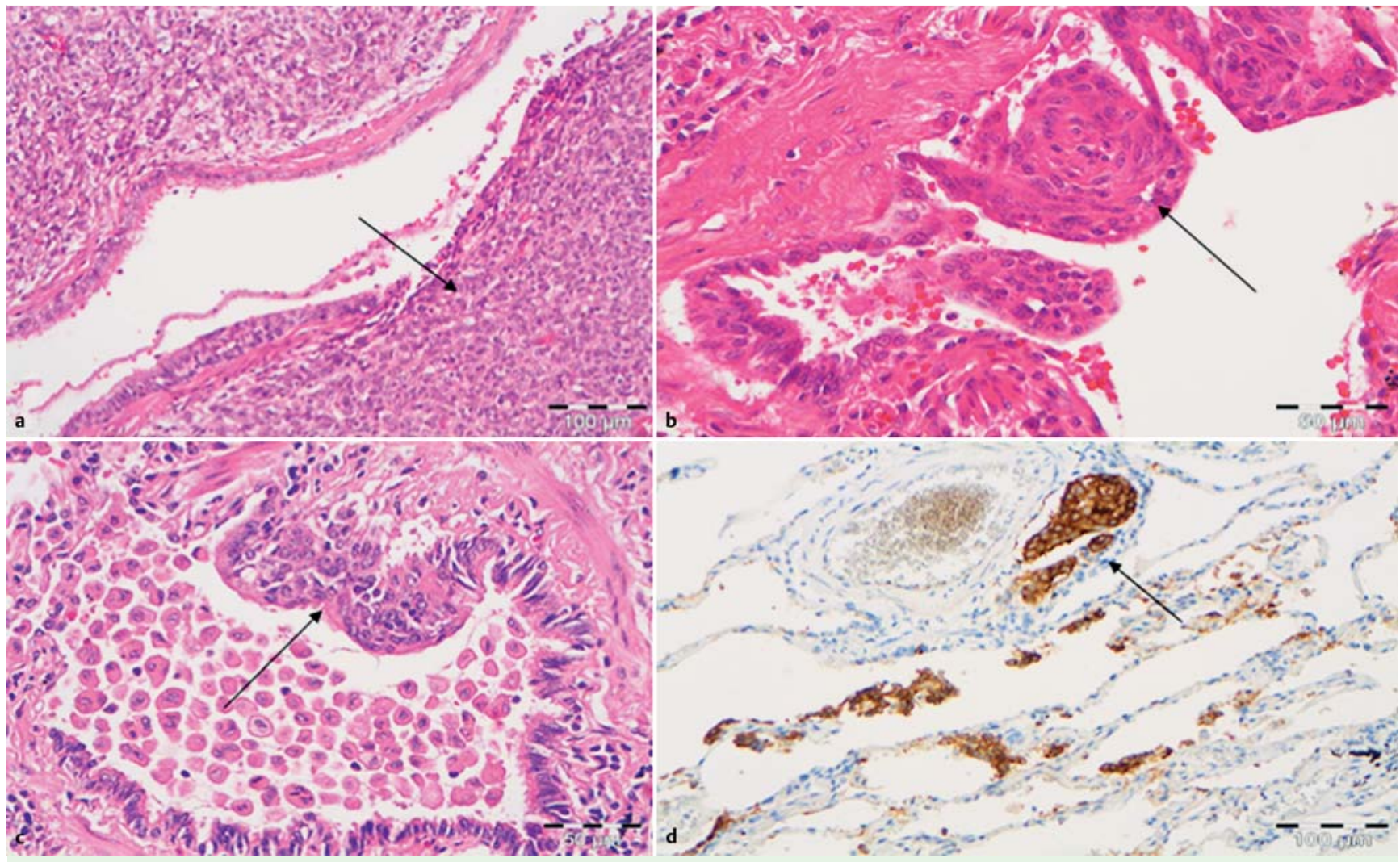

Abb.1 a Atypischer Bronchuskarzinoidtumor (Ki67 22\%) Hämatoxylin-Eosin-Färbung, mikroskopische Vergrößerung 20-fach. b Typischer Karzinoidtumor (Ki67 < 1\%) Hämatoxylin-Eosin-Färbung; mikroskopische Vergrößerung: 20-fach. c Neuroendokrine Zellcluster unter dem Bronchialepithel; Hämatoxylin-EosinFärbung; mikroskopische Vergrößerung: 40-fach. d Immunhistochemische Färbung zu c: Synaptophysin positive Färbung der neuroendokrinen Zellverbände; mikroskopische Vergrößerung: 20-fach.

\section{Diagnostik}

$\nabla$

Die Diagnose von multiplen neuroendokrinen Lungentumoren kann oft schwierig sein, oft liegen Jahrzehnte zwischen Beginn der klinischen Beschwerden und der Diagnose [9]. Die konventionelle Thoraxaufnahme ist bei der Diagnosestellung wenig hilfreich, da sich in der Regel bis auf eine mögliche geringe Bronchialwandverdickung keine Auffälligkeiten zeigen [17]. Das typische Erscheinungsbild multifokaler neuroendokriner Lungentumoren in der Computertomografie des Thorax sind multiple Lungenherde verschiedener Größe. Bei 7 von 11 Patienten mit einem diagnostizierten multifokalen neuroendokrinen Lungentumor lag ein solcher Befund vor. Zusätzlich zeigten sich im Lungenparenchym eine mosaikartige Lungenparenchymzeichnung und Bronchuswandverdickungen oder Bronchiektasien $[4,9,18]$. Differenzialdiagnostisch muss an eine pulmonale Metastasierung eines Primärtumors oder auch an interstitielle Lungenerkrankungen gedacht werden (siehe $\bullet$ Abb.2).

Ein hochauflösendes CT des Thorax (HR-CT) ist indiziert, um eine begleitende Bronchiolitis zu diagnostizieren [9]. Als minimale Bedingung für die Diagnose von multifokalen neuroendokrinen Lungentumoren sollten neben multifokalen Zellhyperplasien mindestens drei oder mehr Tumorlets/Karzinoidtumoren in beiden Lungen vorliegen [19]. Daher ist meist eine thorakoskopisch entnommene Lungenbiopsie zur Diagnosesicherung notwendig. Die chirurgische Lungenbiopsie gilt als der diagnostische Goldstandard [20]. In der pathologischen Untersuchung des entnommenen Lungenparenchymes zeigen sich oft multiple neuroendo-

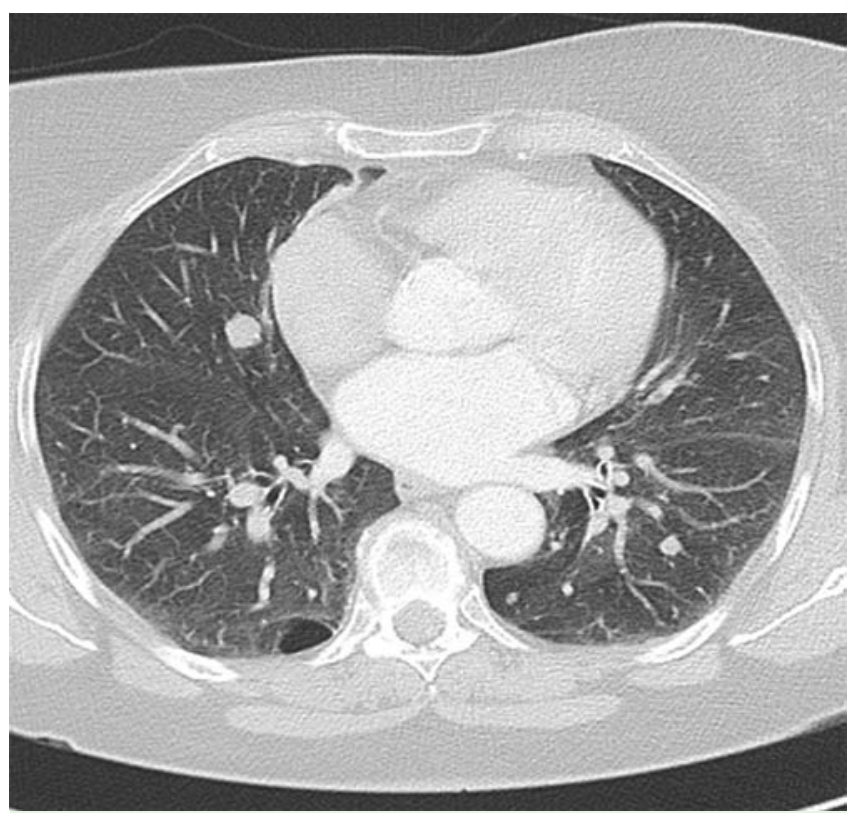

Abb.2 Typische Computertomografie des Thorax mit dem Befund eines MFLNET, das auf beiden Seiten multiple pulmonale Herde verschiedener Größe zeigt.

krine Zellhyperplasien neben Tumorlets und Karzinoidtumoren verschiedener Größe. In seltenen Fällen kann die Diagnose auch durch eine transbronchiale Biopsie gelingen [5]. 
Es wurden zusätzlich Assoziationen der DIPNECH mit Endokrinopathien beschrieben: Akromegalie, ACTH-Produktion und MEN-1-Syndrom [12].

Ein sekundäres Cushing-Syndrom aufgrund einer ektopen ACTHProduktion ist sehr selten in Verbindung mit multifokalen neuroendokrinen Lungentumoren (bisher sind nur 5 Fälle publiziert). Der Grund für eine intermittierende ACTH-Produktion ist unklar [21]. Fessler et al. beschrieben einen Fall von multifokalen neuroendokrinen Lungentumoren und einer Produktion von GHRH und ACTH bei einer gleichzeitig vergrößerten Hypophyse [11].

Fallbericht 2: Bei einer 63-jährigen Patientin kam es seit einem halben Jahr zu einer progredienten respiratorischen Verschlechterung mit rezidivierenden Dekompensationen. Intermittierend musste eine nicht-invasive sowie eine invasive Beatmung durchgeführt werden. Eine Computertomografie des Thorax zeigte multiple beidseitige Lungenherde in allen Lungenlappen mit einer Größe von $<1 \mathrm{~cm}$. Atypische Lungenbiopsien im Bereich des rechten Ober- und Mittellappens zeigten histologisch für das Oberlappenpräparat mindestens 10 bis $0,6 \mathrm{~cm}$ große typische Bronchuskarzinoide (Ki67 Proliferationsrate 1\%), Tumorlets und neuroendokrine Zellhyperplasien und für das Mittellappenpräparat mindestens 24 bis $0,7 \mathrm{~cm}$ große Karzinoidtumore (Ki 67 Proliferationsrate 1\%); Tumorlets und zahlreiche neuroendokrine Zellhyperplasien. Zusätzlich zeigte sich eine ektope ACTHProduktion (99,2 pg/ml; Norm bis $46 \mathrm{pg} / \mathrm{ml}$ ). Bei einem Dexamethasonhemmtest war das ACTH nicht supprimierbar. In der Bildgebung des Schädels zeigte sich keine Vergrößerung der Hypophyse. Ein MEN-1-Syndrom wurde ausgeschlossen.

Gorsthein wertete die PET-CT-Befunde von 11 Patienten (mittleres Alter 62,8 Jahre, alle weiblich) mit multifokalen neuroendokrinen Lungentumoren aus. In $91 \%$ der Fälle zeigte das GADOTATOC PET CT einen sehr hohen Uptake [22].

Es gibt bislang keinen molekularen oder genetischen Marker, um eine DIPNECH von einer reaktiven neuroendokrinen Zellpopulation zu unterscheiden [23]. Huo untersuchte 22 Fälle einer DIP$\mathrm{NECH}$ mit Tumorlets. Immunhistochemisch zeigten sich $\mathrm{CgA}$, Syn, AE1/AE3, TTF1, CD 56 positiv. Der Ki67 Proliferationsindex lag bei $<2 \%$ [24]. Zusätzlich ließ sich bei den DIPNECHs eine ausgeprägte EGFR-Expression nachweisen [25].

\section{Behandlung}

$\nabla$

Zeigt die Bildgebung Lungenherde $<1 \mathrm{~cm}$ Größe bei asymptomatischen Patienten, so wird ein primär abwartendes Beobachten mit jährlicher Kontrollbildgebung empfohlen. Wachsen im Verlauf die Läsionen $>1 \mathrm{~cm}$, so sollten sie chirurgisch entfernt werden $[2,5,6,8]$.

Liegen multifokale Karzinoidtumoren mit einem möglicherweisen Befall der mediastinalen Lymphknoten vor, so sollte eine mediastinale Lymphadenektomie zusätzlich zur Resektion der Herde durchgeführt werden. Pro Seite sollten alle Karzinoidtumoren im Gesunden entfernt werden [26].

Fallbericht 3: Bei einer 69-Jährigen wurde nach einem Verkehrsunfall eine Computertomografie des Thorax durchgeführt. Diese zeigte beidseitige Lungenrundherde bis zu $1 \mathrm{~cm}$. Die Patientin war vor dem Unfall völlig beschwerdefrei und hatte nie geraucht. Eine transbronchiale Biopsie erbrachte keinen wegweisenden Befund. Im Rahmen einer Thorakoskopie wurden zwei Lungenherde atypisch reseziert. Beide Lungenherde waren histologisch typische Karzinoidtumoren (Ki67 Proliferationsrate 1\%). Da die Anzahl der Lungenherde in der Bildgebung begrenzt war, entschlossen wir uns zur beidseitigen offenen Resektion aller Herde. Auf der linken Seite wurden insgesamt acht Lungenherde (mittlere Größe: 0,45cm, Range: 0,2-0,9cm, Ki67 Proliferationsrate $1 \%)$ atypisch unter Einsatz eines diodengepumpten Nd:YAGLasers LIMAX ${ }^{\circledR} 120$ (Firma Gebrüder Martin \& Co KG, Tuttlingen, Germany) im Gesunden entfernt (siehe Abb.3). Auf der rechten Seite wurden 6 Lungenherde (mittlere Größe: 0,35 cm; Range: 0,1-0,9 cm; Ki67 Proliferationsrate: 1\%) ebenfalls atypisch mit dem Laser im Gesunden entfernt. Auf beiden Seiten wurde eine radikale mediastinale Lymphadenektomie durchgeführt, kein mediastinaler Lymphknoten war tumorbefallen. In der Nachuntersuchung zwei Jahre nach den Operationen zeigten sich in der Computertomografie des Thorax keine neuen Lungenherde. Der Patientin geht es sehr gut.

Patienten, die eine klinische Symptomatik mit Verschlechterungstendenz aufweisen, sollten mit Corticosteroiden behandelt werden $[9,27]$. Steroide können die Lungenfunktion verbessern und die sich über einen längeren Zeitraum entwickelnde Lungenfibrose abmildern [5].

Insbesondere wenn keine chirurgische Option zur Entfernung der Karzinoide besteht, sollten Somatostatin-Analoga oder Interferon alpha zur Behandlung eingesetzt werden [22,28].

Es gibt keine Evidenz für den Einsatz von Chemotherapeutika bei der Behandlung multifokaler neuroendokriner Lungentumoren [12,27].

Eine Peptidrezeptorradiotherapie mit Verwendung radioaktiv markierter Somatostatinanaloga ist bei progredienten metastasierten neuroendokrinen Tumoren als therapeutische Option etabliert, der Stellenwert bei nicht-resektablen Bronchuskarzinoiden und DIPNECH ist derzeit unklar [22].

Zhou berichtete über zwei Patienten, die aufgrund multipler neuroendokriner Lungentumoren mit einer stark eingeschränkten Lungenfunktion bei Nichtansprechen der konservativen Therapie doppellungentransplantiert wurden. Die Lungentransplantation erschien in dieser Situation als eine gute Therapieoption $[22,29]$.

\section{Prognose}

Die Prognose der DIPNECH ist gut, nach 5 Jahren leben noch $83 \%$ der Betroffenen [6,9]. Carr et al. berichteten über den Verlauf von 30 Patienten mit einer DIPNECH. 26\% der Patienten zeigten innerhalb von zwei Jahren einen Abfall der FEV1 von 10\%. 44\% der Patienten zeigten eine konstriktive Bronchiolitis in der pathologischen Untersuchung [30]. Unabhängig von der klinischen Manifestationsform ist der Krankheitsverlauf meist über Jahre stabil, zum Teil lediglich durch eine Obstruktion limitiert [8]. Falkenstern-Ge beschrieb den Fall eines 69-jährigen Nichtrauchers mit einer bekannten DIPNECH, dessen Lungenherde, was Anzahl, Größe und Form betrifft, sich über einen Zeitraum von 7 Jahren in der Bildgebung nicht veränderten [28]. Fälle mit einem stabilen Verlauf der Erkrankung über 14 Jahre nach Erstdiagnose wurden beschrieben [31]. Gesicherte Daten zur Prognose von multifokalen neuroendokrinen Lungentumoren gibt es nicht. Es ist anzunehmen, dass sie sich an den Daten, die für eine DIPNECH bekannt sind, orientieren. 


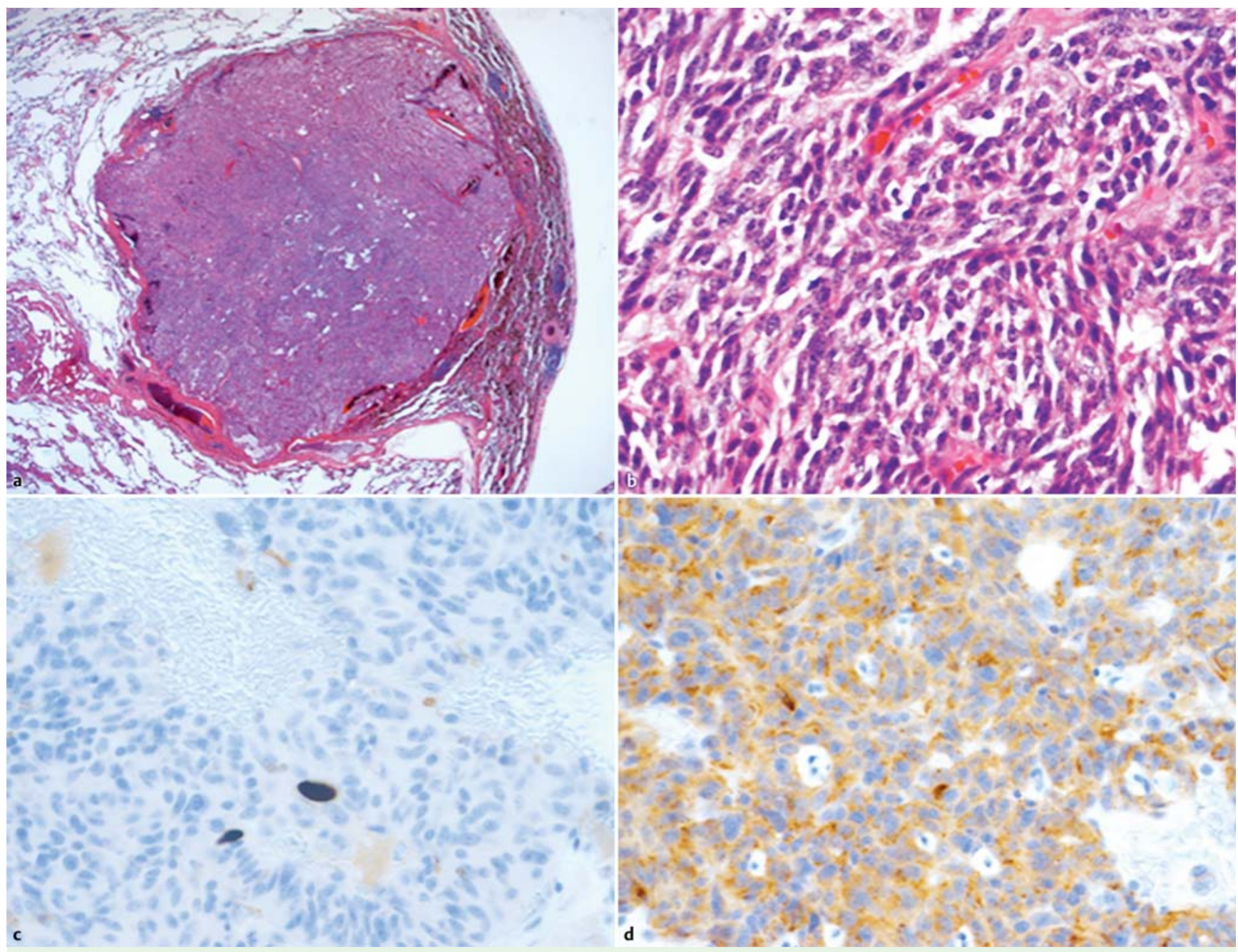

Abb.3 a Subpleuraler typischer Karzinoidtumor; Hämatoxylin-Eosin-Färbung; mikroskopische Vergrößerung: 12,5-fach. b Gleicher Tumor nur bei 400-facher Vergrößerung: monomorphes Zellbild, keine Mitosen; Hämatoxylin-Eosin-Färbung. c Ki67 Proliferationsmarker < 1\%; Immunhistochemische Färbung; mikroskopische Vergrößerung: 400-fach. d Der neuroendokrine Marker Chromogranin A ist positiv (immunhistochemische Färbung; mikroskopische Vergrößerung: 400-fach).

Therapiealgorithmus zur Behandlung multifokaler neuroendokriner Lungentumoren (siehe $\bullet$ Abb.4)

Das von uns vorgeschlagene therapeutische Vorgehen bei diagnostizierten multifokalen pulmonalen neuroendokrinen Lungentumoren orientiert sich an den klinischen Beschwerden des Patienten. Stufenweise werden die medikamentösen Behandlungen dem jeweiligen eingetretenen Therapieerfolg angepasst. In jeder Phase der Behandlung sollte überprüft werden, ob pulmonale Herde, die größer als $1 \mathrm{~cm}$ sind, nicht chirurgisch reseziert werden sollten.

Werden bei einem Patienten multifokale neuroendokrine Lungentumoren diagnostiziert, so ist die erste Frage nach der bestehenden klinischen Symptomatik wie z.B. Husten oder Luftnot. Liegt diese nicht vor und sind auf dem HR-CT des Thorax Lungenherde zu sehen, die $>1 \mathrm{~cm}$ sind, so kann der Chirurg eine Resektion prüfen. Ziel der Operation ist hier, alle Herde im Gesunden zu resezieren [12,22]. Da mehrere Herde vorliegen, sollten atypische Resektionen durchgeführt werden. Zusätzlich zu den pulmonalen Resektionen sollte eine radikale mediastinale Lymphadenektomie durchgeführt werden. Nach der erfolgten Resektion sollte in 6-monatigen Abständen eine Kontrolluntersuchung durchgeführt werden. Kommt aufgrund der Bildgebung eine chi- rurgische Intervention nicht in Frage, so sollte zunächst aufgrund der Beschwerdefreiheit eine Kontrolluntersuchung in 6 Monaten durchgeführt werden [22]. Ist der Patient symptomatisch, so sollte zunächst eine inhalative Therapie (z.B. mit SABA, LABA, ICS) versucht werden [12]. Bei Rückgang der Beschwerden oder sogar Beschwerdefreiheit sollte die Resektabilität der pulmonalen Herde geprüft werden. Tritt keine Besserung der klinischen Beschwerden auf, so sollte dann eine Steroidtherapie durchgeführt werden [12]. Ist hierunter eine klinische Verbesserung der Symptome zu erreichen, so sollte erneut die Möglichkeit einer chirurgischen Resektion geprüft werden. Bei Beschwerdepersistenz oder -progredienz sollte eine Targeted-Therapie (Somatostatinanaloga, Everolimus, Sunitinib) oder eine Chemotherapie (z.B. Temozolomid) geprüft werden [32]. Bei Ansprechen der Therapie sollte erneut die Möglichkeit einer chirurgischen Resektion überprüft werden. Versagen alle konservativen Therapiemöglichkeiten bei fortschreitendem Krankheitsprogress, so kann in dieser Situation eine Doppellungentransplantation in Erwägung gezogen werden [29]. 


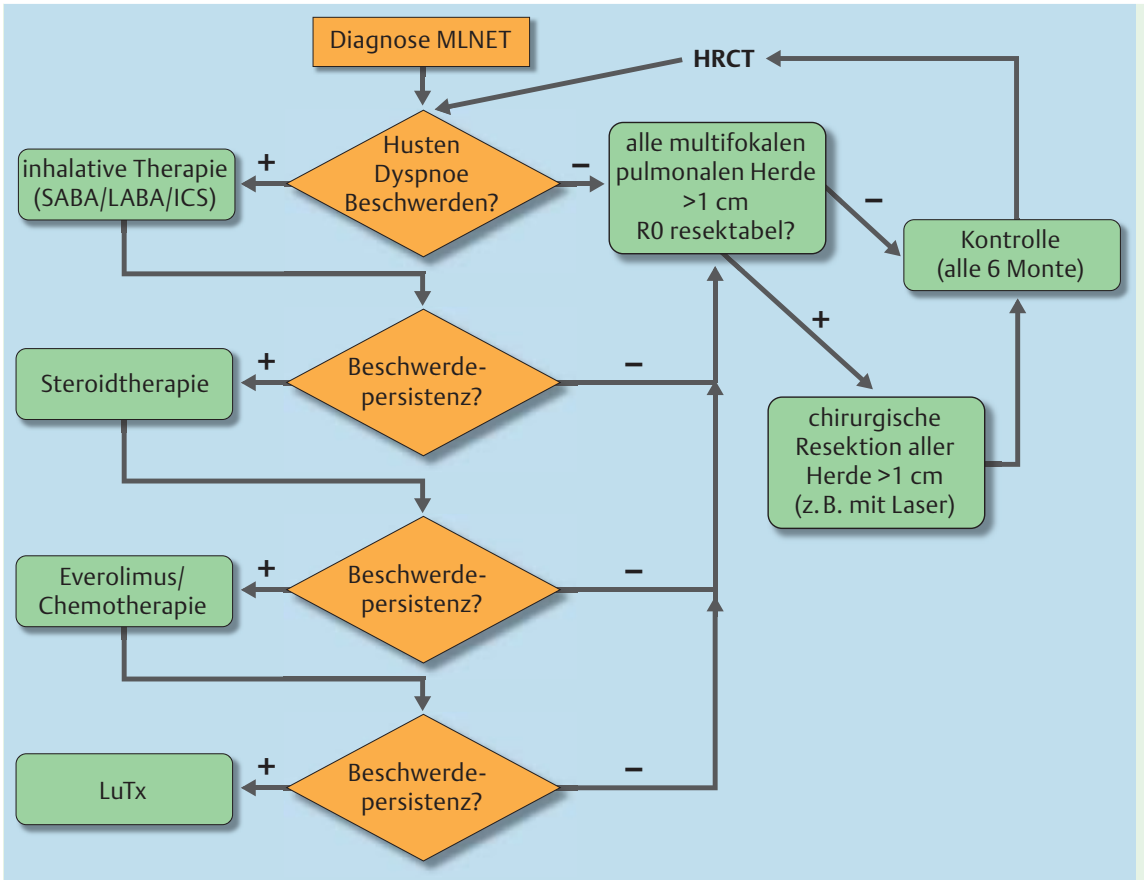

Abb.4 Stufenweises therapeutisches Vorgehen bei multifokalen neuroendokrinen Lungentumoren.

\section{Zusammenfassung}

Selten sieht sich der Kliniker mit multifokalen neuroendokrinen Lungentumoren konfrontiert. Charakteristisch in der Bildgebung ist meist eine Mischung aus pulmonalen Herden unterschiedlicher Größe. Es ist anzunehmen, dass sich die multifokalen neuroendokrinen Lungentumoren aus diffusen neuroendokrinen Zellhyperplasien, die durch ihre Größenzunahme zu Lungenkarzinoidtumoren werden, entwickeln. Die Diagnose wird in den meisten Fällen durch eine chirurgische Lungenbiopsie gestellt. Die Behandlung orientiert sich nach dem klinischen Beschwerdebild. Bei asymptomatischen Patienten mit multifokalen Lungenherden $<5 \mathrm{~mm}$ kann durchaus nur eine halbjährliche Kontrolluntersuchung ohne spezifische Therapie erfolgen. Bei symptomatischen Patienten mit einem deutlichen Krankheitsprogress stehen verschiedenste Therapieoptionen von der inhalativen Therapie über eine Steroidtherapie bis hin zu Angiogenesehemmern (z.B. Everolimus) zur Verfügung.

\section{Interessenkonflikt}

$\nabla$

Die Autoren geben an, dass kein Interessenkonflikt besteht.

\section{Institute}

Klinik für Viszeral-, Thorax- und Gefäßchirurgie, Uniklinik Gießen und Marburg (UKGM), Standort Marburg

2 Klinik für Pneumologie, Uniklinik Gießen und Marburg (UKGM), Standort Marburg

Klinik für Gastroenterologie, Uniklinik Gießen und Marburg (UKGM), Standort Marburg

${ }^{4}$ Abteilung für Pathologie, Uniklinik Gießen und Marburg (UKGM),

Standort Marburg

${ }^{5}$ Institut für Pathologie und Zytologie, Überregionale Gemeinschaftspraxis, Wetzlar

\section{Literatur}

1 Schnabel PA, Junker K. [Neuroendocrine tumors of the lungs. From small cell lung carcinoma to diffuse idiopathic pulmonary neuroendocrine cell hyperplasia]. Der Pathologe 2014; 35: 557-564

2 Patel C, Tirukonda P, Bishop R et al. Diffuse idiopathic pulmonary neuroendocrine cell hyperplasia (DIPNECH) masquerading as metastatic carcinoma with multiple pulmonary deposits. Clin Imaging 2012; 36: $833-836$

3 Killen $\mathrm{H}$. DIPNECH presenting on a background of malignant melanoma: new lung nodules are not always what they seem. BMJ case reports 2014; DOI 10.1136/bcr-2014-203667

4 Chassagnon G, Favelle 0 , Marchand-Adam S et al. DIPNECH: when to suggest this diagnosis on CT. Clin Radiol 2015; 70: 317-325

5 Al-Ayoubi AM, Ralston JS, Richardson SR et al. Diffuse pulmonary neuroendocrine cell hyperplasia involving the chest wall. The Annals of thoracic surgery 2014; 97: $333-335$

6 Walker CM, Vummidi D, Benditt JO et al. What is DIPNECH? Clin Imaging 2012; 36: 647-649

7 Mireskandari M, Abdirad A, Zhang $Q$ et al. Association of small foci of diffuse idiopathic pulmonary neuroendocrine cell hyperplasia (DIPNECH) with adenocarcinoma of the lung. Pathol Res Pract 2013; 209: $578-584$

8 Pizarro $C$, Zhou H, Kader $R$ et al. [Diffuse idiopathic pulmonary neuroendocrine cell hyperplasia (DIPNECH) in a 67-year-old patient with chronic coughing]. Deutsche medizinische Wochenschrift (1946) 2014; 139: $1245-1248$

9 Benson RE, Rosado-de-Christenson ML, Martinez-jimenez S et al. Spectrum of pulmonary neuroendocrine proliferations and neoplasms. Radiographics 2013; 33: 1631 - 1649

10 Coletta EN, Voss LR, Lima MS et al. Diffuse idiopathic pulmonary neuroendocrine cell hyperplasia accompanied by airflow obstruction. Jornal brasileiro de pneumologia: publicacao oficial da Sociedade Brasileira de Pneumologia e Tisilogia 2009; 35: 489-494

11 Fessler MB, Cool CD, Miller YE et al. Idiopathic diffuse hyperplasia of pulmonary neuroendocrine cells in a patient with acromegaly. Respirology (Carlton, Vic) 2004; 9: 274-277

12 Nassar AA, Jaroszewski DE, Helmers RA et al. Diffuse idiopathic pulmonary neuroendocrine cell hyperplasia: a systematic overview. Am J Respir Crit Care Med 2011; 184: 8-16

13 Reyes $L J$, Majo J, Perich $D$ et al. Neuroendocrine cell hyperplasia as an unusual form of interstitial lung disease. Respir Med 2007; 101: $1840-1843$ 
14 Gosney JR, Williams IJ, Dodson AR et al. Morphology and antigen expression profile of pulmonary neuroendocrine cells in reactive proliferations and diffuse idiopathic pulmonary neuroendocrine cell hyperplasia (DIPNECH). Histopathology 2011; 59: 751 - 762

15 Ruffini E, Bongiovanni M, Cavallo A et al. The significance of associated pre-invasive lesions in patients resected for primary lung neoplasms. Eur J Cardiothoracic Surg 2004; 26: 165-172

16 Miller RR, Muller NL. Neuroendocrine cell hyperplasia and obliterative bronchiolitis in patients with peripheral carcinoid tumors. Am J Surg Pathol 1995; 19: 653-658

17 Greiner B, Schulz C, Pfeifer M et al. [A 74-year-old female patient with histologically proven carcinoid of the lungs and pulmonary mosaic pattern]. Der Radiologe 2009; 49: 538-541

18 Foran PJ, Hayes SA, Blair DJ et al. Imaging appearances of diffuse idiopathic pulmonary neuroendocrine cell hyperplasia. Clin Imaging 2015; 39: $243-246$

19 Marchevsky AM, Wirtschafter E, Walts AE. The spectrum of changes in adults with multifocal pulmonary neuroendocrine proliferations: what is the minimum set of pathologic criteria to diagnose DIPNECH? Hum Pathol 2015; 46: 176-181

20 McGuire AL, Maziak DE, Sekhon HS. Diffuse intrapulmonary neuroendocrine cell hyperplasia. Can Respir J 2013; 20: 406-408

21 Cameron CM, Roberts F, Connell J et al. Diffuse idiopathic pulmonary neuroendocrine cell hyperplasia: an unusual cause of cyclical ectopic adrenocorticotrophic syndrome. Br J R 2011; 84: e14-e17

22 Gorshtein A, Gross DJ, Barak D et al. Diffuse idiopathic pulmonary neuroendocrine cell hyperplasia and the associated lung neuroendocrine tumors: clinical experience with a rare entity. Cancer 2012; 118: $612-619$

23 Lantuejoul S, Salameire D, Salon C et al. Pulmonary preneoplasia-sequential molecular carcinogenetic events. Histopathology 2009; 54: $43-54$
24 Huo Z, Shi XH, Cui QC et al. [Pulmonary neuroendocrine cell hyperplasia and tumorlets in bronchiectasis: a clinicopathologic study of $22 \mathrm{ca}-$ ses with review of literature]. Zhonghua bing li xue za zhi Chinese journal of pathology 2012; 41: 525-529

25 Kuhnen C, Winter BU. [EGFR-expression in pulmonary neuroendocrine cell hyperplasia]. Der Pathologe 2006; 27: 147-151

26 Ferolla $P$, Daddi $N$, Urbani $M$ et al. Tumorlets, multicentric carcinoids, lymph-nodal metastases, and long-term behavior in bronchial carcinoids. J Thorac Oncol 2009; 4: 383-387

27 Montoro Zulueta FJ, Martinez Prieto M, Verdugo Cartas MI et al. Diffuse idiopathic pulmonary neuroendocrine cell hyperplasia with multiple synchronous carcinoid tumors. Archivos de bronconeumologia 2012; 48: $472-475$

28 Falkenstern-Ge RF, Kimmich M, Friedel $G$ et al. Diffuse idiopathic pulmonary neuroendocrine cell hyperplasia: 7-year follow-up of a rare clinicopathologic syndrome. J Cancer Res Clin Oncol 2011; 137: $1495-1498$

29 Zhou H, Ge Y, Janssen B et al. Double lung transplantation for diffuse idiopathic pulmonary neuroendocrine cell hyperplasia. J Bronchology Interv Pulmonol 2014; 21: 342 - 345

30 Carr LL, Chung JH, Duarte Achcar R et al. The clinical course of diffuse idiopathic pulmonary neuroendocrine cell hyperplasia. Chest 2015 147: $415-422$

31 Johney EC, Pfannschmidt J, Rieker RJ et al. Diffuse idiopathic pulmonary neuroendocrine cell hyperplasia and a typical carcinoid tumor. J Thorac Cardiovasc Surgery 2006; 131: 1207-1208

$32 \mathrm{Oba}$ H, Nishida K, Takeuchi S et al. Diffuse idiopathic pulmonary neuroendocrine cell hyperplasia with a central and peripheral carcinoid and multiple tumorlets: a case report emphasizing the role of neuropeptide hormones and human gonadotropin-alpha. Endocrine pathology 2013; $24: 220-228$ 